\title{
Mechanism of surface modification in the plasma-surface interaction in electrical arcs
}

\author{
H. Timko \\ CERN, CH-1211 Genève 23, Switzerland \\ and Helsinki Institute of Physics and Department of Physics, University of Helsinki, P.O. Box 43, FIN-00014 Helsinki, Finland \\ F. Djurabekova and K. Nordlund* \\ Helsinki Institute of Physics and Department of Physics, University of Helsinki, P.O. Box 43, FIN-00014 Helsinki, Finland \\ L. Costelle \\ Department of Physics, University of Helsinki, P.O. Box 43, FIN-00014 Helsinki, Finland \\ K. Matyash and R. Schneider \\ Max-Planck-Institut für Plasmaphysik, EURATOM Association, Wendelsteinstrasse 1, D-17491 Greifswald, Germany
}

\begin{abstract}
A. Toerklep, G. Arnau-Izquierdo, A. Descoeudres, S. Calatroni, M. Taborelli, and W. Wuensch CERN, CH-1211 Genève 23, Switzerland

(Received 23 December 2009; revised manuscript received 22 February 2010; published 25 May 2010)
\end{abstract}

\begin{abstract}
Electrical sparks and arcs are plasma discharges that carry large currents and can strongly modify surfaces. This damage usually comes in the form of micrometer-sized craters and frozen-in liquid on the surface. Using a combination of experiments, plasma and atomistic simulation tools, we now show that the observed formation of deep craters and liquidlike features during sparking in vacuum is explained by the impacts of energetic ions, accelerated under the given conditions in the plasma sheath to kiloelectron volt energies, on surfaces. The flux in arcs is so high that in combination with kiloelectron volt energies it produces multiple overlapping heat spikes, which can lead to cratering even in materials such as $\mathrm{Cu}$, where a single heat spike normally does not.
\end{abstract}

DOI: 10.1103/PhysRevB.81.184109

PACS number(s): 79.20.Rf, 52.80.Mg, 52.80.Vp, 61.80.Jh

\section{INTRODUCTION}

Sparks and arcs are well familiar from everyday life, occurring in a wide array of situations ranging from the pleasant, such as fireplaces, the practical, like arc welding and spark plugs for cars, to the spectacular, like lightning in the sky. ${ }^{1-3}$ Arcs are known to be electrical discharges between two electrodes that can occur in gas or vacuum..$^{1,3,4}$ The end result of arcs is surface damage that is often harmful, as in the case of linear collider components, ${ }^{5}$ fusion reactors, ${ }^{6}$ or spark plugs for cars, ${ }^{7}$ but is also utilized for electrical discharge machining of materials. ${ }^{8}$ The science behind arcing is surprisingly poorly understood, considering how widely they occur. In particular, several alternative mechanisms have been proposed to explain the surface modifications associated with sparking and arcing, such as thermionic electron emission, photoinduced ionization, sputtering, evaporation, and oxide removal. ${ }^{1,7,9,10}$ It still remains unclear which ones are truly active and what is their range of applicability. ${ }^{7}$ Understanding the mechanism is crucial for guiding the choice of materials for new high-gradient accelerator concepts and fusion reactors.

The plasma-surface interaction in $\operatorname{arcs}^{11}$ is typically observed to produce either single or multiple overlapping craters on surfaces and features which clearly resemble liquid blobs that have emanated from the crater and been frozen-in on the surface (for typical examples see Figs. 1 and 2). Formation of craters is, on the other hand, also observed on planets and moons due to asteroids, ${ }^{12}$ on materials hit by high-velocity projectiles, ${ }^{13}$ and surprisingly also on the atomic scale due to single accelerated ions and atom clusters hitting surfaces. ${ }^{14,15}$ Quite recently all these ranges of cratering were linked together, when it was shown that the craters produced by atom clusters of sizes of roughly 10000 or more atoms produce cratering due to the same pressurerelated mechanism as the macroscopic impactors. ${ }^{16}$ When this size has been reached, the crater formation in the atomistic simulations follows the same scaling law as the macroscopic impacts over about 35 orders of magnitude in size scale $^{16}$ and exhibits all the central features of experimental cratering, such as splashing, crown, and corona formation. ${ }^{17-20}$ The single-atom cratering, by contrast, is now well established to be caused by liquid flow, associated with the formation of a "heat spike," i.e., hot pressurized matter formed after high-energy incoming ions are thermalized in a sequence of ballistic collisions. ${ }^{15,16,21,22}$ Although both the

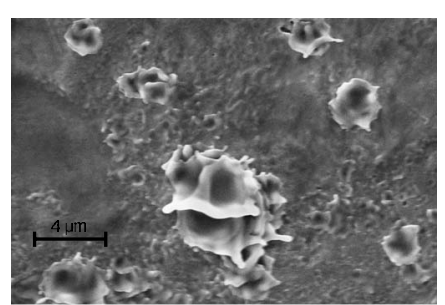

(a)

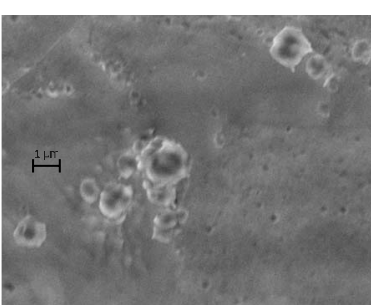

(b)
FIG. 1. Craters produced during arcing at the dc arc setup at CERN. (a) and (b) show SEM images of two different sparking events. The experiments show craters of similar shape in a wide variety of sizes in the range $\sim 0.1-10 \mu \mathrm{m}$, justifying the comparison with smaller simulated craters. 

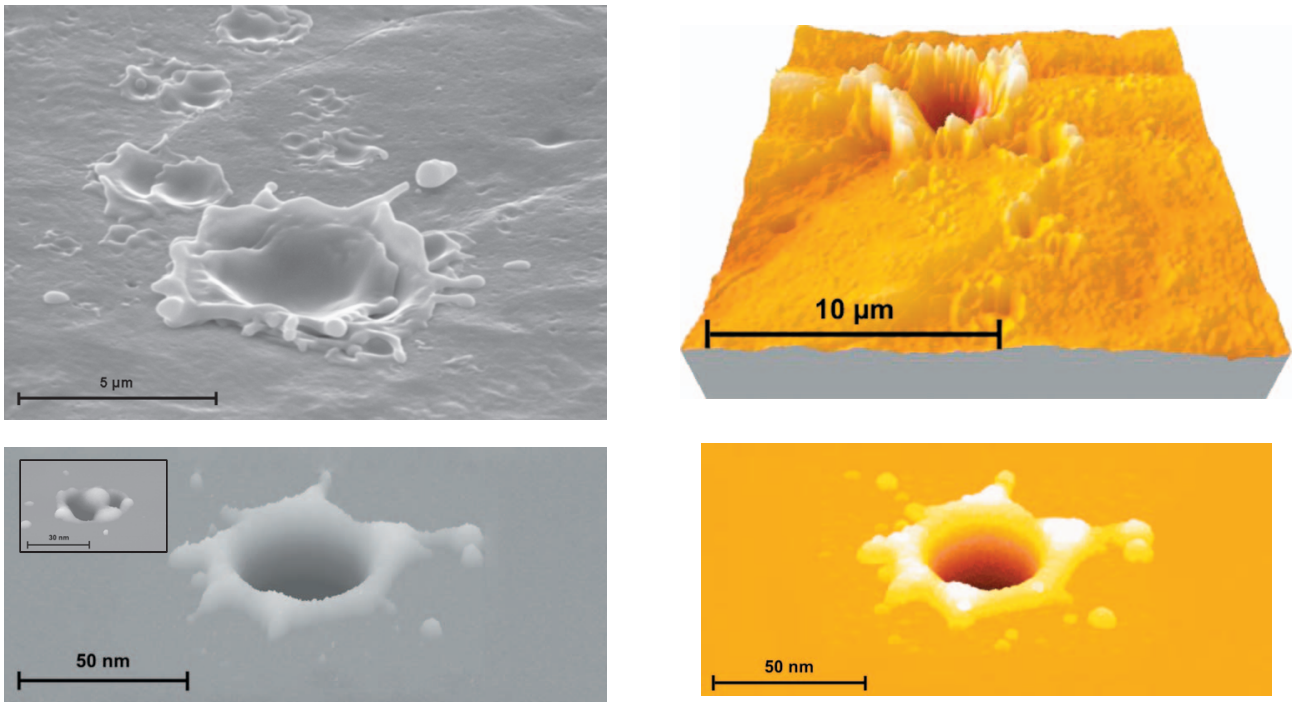

FIG. 2. (Color) Comparison of experimental and simulated craters. Top left: tilted SEM experimental image of a few nearby craters. Top right: tilted AFM experimental image of the same experimental crater. Bottom left: tilted MD simulated image of a single crater. Bottom right: MD simulated image of the same simulated crater. In the simulated case the total-energy deposition was $4.34 \mathrm{keV} / \mathrm{nm}^{2}$. The inset in the bottom left part shows a complex double crater from the MD simulations.

macroscopic and atom-induced craters bear a clear visual resemblance to the craters produced by arcing, the possible relation between arc and impact cratering mechanisms has not been established.

\section{METHODS}

In the work presented, we are modeling vacuum arcs, i.e., arcs which occur between two solid electrodes under ultrahigh-vacuum (UHV) conditions.

\section{A. Experiments}

Experiments on cratering were carried out in an UHV chamber using the direct-current (dc) arcing setup at CERN presented in Refs. 23 and 24. Briefly, the experimental setup used consists of a rounded rod (anode) and a plane sample (cathode). The $\mathrm{Cu}$ cathode and anode were separated by $20 \mu \mathrm{m}$, and a voltage of up to $10 \mathrm{kV}$ was placed between the electrodes. The voltage was obtained by charging a capacitor to a given high voltage, then disconnecting it from the power supply, and finally connecting it to the electrodes. This lead to electric fields of up to $\sim 500 \mathrm{MV} / \mathrm{m}$, which induced arcing between the electrodes.

For the current paper a series of ten single sparks on the same sample, well separated from each other, was generated. The result was a complex central crater produced by multiple impact of the plasma on the surface due to random movement of the cathode spot, as expected from the literature. ${ }^{2}$ Adjacent to this main crater we observed single craters of varying size produced by single or a couple of overlapping arc-surface interactions, see Fig. 1. These were chosen for the further experimental and computational analysis.

The surface morphology of the cratering was analyzed by a LEO 430 scanning electron microscope (SEM) operated with a voltage of $20 \mathrm{kV}$ at CERN, and with an Autoprobe CP
Research (Thermo Microscope) atomic force microscope (AFM) at the University of Helsinki. The AFM tip was a tungsten-carbide-coated silicon cantilever from MikroMasch (CSC11/W2C) with a cone angle of $40^{\circ}$ and probe tip radius of curvature below $35 \mathrm{~nm}$. Since this is much less than the micrometer-scale widths of the crater, tip convolution was not a serious issue.

\section{B. Simulations}

The plasma formation under corresponding conditions was simulated using the particle-in-cell (PIC) method ${ }^{25,26}$ in which the kinetics of so-called "superparticles" (each of them representing many real particles, that in the current case were electrons, $\mathrm{Cu}$ neutrals, or $\mathrm{Cu}^{+}$ions), moving in selfconsistent fields, is calculated on the grid with Maxwell's equations. The particle collisions are handled by Monte Carlo collision (MCC) routines, which randomly change particle velocities according to the actual collision dynamics. As in the experimental setup the high electric field is concentrated to a small area on both the anode and the cathode, the system can be approximated by two parallel plane electrodes, with a homogeneous external electric field in between. Hence, the PIC simulations treated the plasma formation between a system of infinite plates, approximating the main stream of the plasma between the electrodes. A PIC-MCC code developed within IPP Greifswald, ${ }^{27}$ which resolves one spatial but three velocity components $(1 \mathrm{~d} 3 \mathrm{v})$, was used for the simulations.

In these PIC simulations, the same conditions as in experiments have been applied. The distance between the electrodes was $20 \mu \mathrm{m}$ with the cathode grounded and the anode powered with $10 \mathrm{kV}$. The three dominating species, electrons, $\mathrm{Cu}$ and $\mathrm{Cu}^{+}$were taken into account. Simulations started from perfect vacuum. A constant rate of electron emission and neutral evaporation was assumed throughout 
all the simulation. The boundary conditions assumed in this simple model were the following: (i) electrons sputter $\mathrm{Cu}$ at the walls with a sputtering yield $Y=0.01$, (ii) $\mathrm{Cu}^{+}$sputter $\mathrm{Cu}$ with $Y=1$, and (iii) $\mathrm{Cu}$ is reflected back. Ions are obtained in the system due to impact ionization collisions. Together with all other relevant collisions (Coulomb collisions, elastic collisions, and charge exchange), these collisions were treated with the MCC scheme, and utilized experimentally measured cross section data. ${ }^{28-30}$ The one-dimensional spatial coordinate and the three velocity components of each particle were followed every time step. The development of the plasma was analyzed through plasma macroquantities (number densities and temperature of each species, as well as electric potential in the system) that are outputted regularly. Also other quantities of interest, such as energy distributions and total flux of each species, can optionally be computed and outputted.

Given the above-mentioned initial and boundary conditions, the plasma develops as follows (see also animation DC.avi in supplementary material ${ }^{31}$ ). First the neutral density builds up in the system since ionization events are rare in the beginning. In addition, charged particles are accelerated in the electric field and therefore leave the system quickly, while neutrals move slowly, so that they can fill the electrode gap. When then at one point the neutral density reaches a critical value that corresponds to a mean-free path $l_{m f p}$ of the electron impact ionization which becomes smaller than the spacing between the electrodes $l_{\text {sys }}$, an avalanche of ionization is reached. This is the "point of no return," since the increased ion flux results in further sputtering, creating even more neutrals in the system, leading to increased ionization, which then again results in an enhanced ion flux, enhanced erosion, and so forth. The ion density builds up, and a quasineutral plasma is formed starting from the anode. The external electric potential is then confined to a small region close to the cathode, the plasma sheath, where the more mobile electrons are decelerated and the ions are accelerated by the external field until an equilibrium flow is reached (through a given difference in electron and ions densities in the sheath). However, the spontaneous arc itself, which is modeled here, never reaches equilibrium, since densities are steadily growing as long as energy is supplied to the system. A detailed description of the plasma simulation model and additional results of the time evolution of the plasma is presented in Ref. 32.

The PIC simulations gave as an output the flux and energy distribution of $\mathrm{Cu}$ ions hitting the cathode after the arc plasma was formed (see Fig. 3). To remove numerical fluctuations, the energy distribution and flux was averaged over a few nanoseconds of the buildup phase of the plasma, after ionization has occurred and a sheath has formed. Therefore, on the time scale of molecular dynamics (MD) surface damage simulations (approximately picosecond), these quantities can be regarded as stationary. The interaction of the ions from the plasma with the $\mathrm{Cu}$ surface was simulated with classical MD (Refs. 21 and 33) methods using the fully parallel PARCAS code developed within the group, utilizing up to 2000 processors and total run times of up to $200000 \mathrm{~h}$ for a single event.

The $\mathrm{Cu}$ interatomic interactions in the MD simulations were described with the well-tested potential by Sabochick

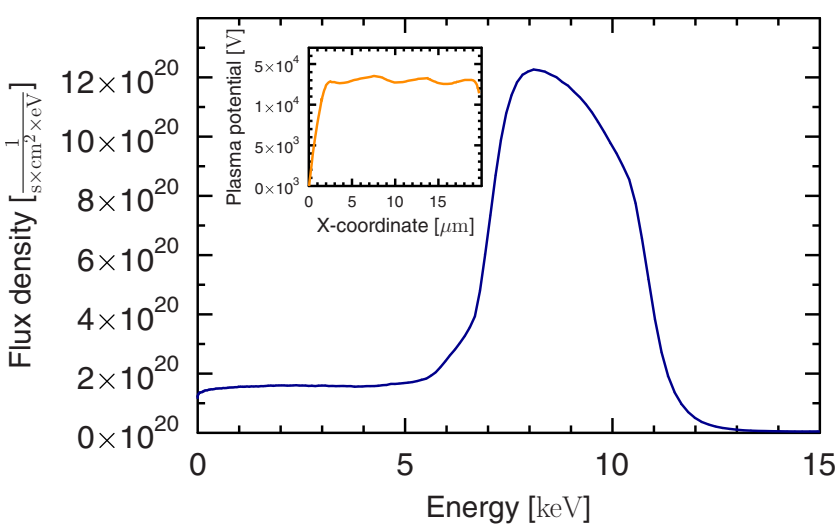

FIG. 3. (Color online) Results from PIC simulations of the development of an arc plasma. The figure shows the total flux of $\mathrm{Cu}$ ions from the plasma against the cathode material. Due to the acceleration in the sheath potential, the energy distribution has a strong maximum around the high kinetic energy/atom of $8 \mathrm{keV}$. The inset shows the distribution of electric potential between the cathode (at $x=0)$ and anode $(x=20 \mu \mathrm{m})$ after the arc plasma has formed. The plasma sheath leads to a strong gradient in the potential near the cathode, leading to the acceleration of $\mathrm{Cu}$ ions toward it.

and Lam. ${ }^{21,34-36}$ The ion flux was modeled selecting the impact times randomly in the Poisson distribution ${ }^{37}$ such that the average flux corresponded to the one simulated with PIC. The energies were generated in a random distribution corresponding exactly to that obtained from the PIC simulations and the impact positions on the surface were generated randomly within a circle of radius $r=3-25 \mathrm{~nm}$. Due to the high flux $\left(\sim 10^{25}\right.$ ions $\left./ \mathrm{cm}^{2} / \mathrm{s}\right)$ from the plasma, ions impinge with time intervals $\Delta t_{0}$ on the order of 1-100 fs, depending on surface area and thus all ions were introduced in the same MD simulation for a given area and dose. To get statistics, 2-5 simulations were carried out with the same area and dose.

To properly deal with high-energy effects, we used simulation methods such as adaptive time steps, energy loss to electrons, and repulsive potentials well established for the simulation of energetic ion interactions with materials. ${ }^{16,21}$ The adaptive time step algorithm previously used in our group $^{38}$ was for the current paper augmented to ensure that there are always at least 3 time steps simulated between the impact of each new ion. To fully contain the heat spikes, we used system sizes of up to 20 million atoms.

To test the notion that the high ion energies are indeed crucial for obtaining complex crater shapes, we also carried out "thermal" runs, modeling a condition where the surface would be heated by equilibrium effects only (low-energy electrons and ions and possibly Joule heating from emitted electrons). In these simulations, in contrast to our main simulations, we did not introduce any energetic ions (i.e., a condition with no accelerating electric sheath potential) which could cause radiation damage. We deposited energy to atoms in the same $r=15 \mathrm{~nm}$ area as in the energetic runs but only in the top $1 \mathrm{~nm}$. This depth was chosen to correspond to energy deposition to the surface atom layers only by lowenergy particles that do not penetrate deep into the sample. 
More specifically, the energy deposition was carried out by giving randomly chosen atoms in the defined volume a kinetic energy of $10 \mathrm{eV}$ to a random direction, to produce on average an even volume distribution of energy deposition. Since $10 \mathrm{eV}$ is below the threshold displacement energy in $\mathrm{Cu}$ (Ref. 39), this energy does not lead to any ballistic collisions but corresponds to a thermal energy deposition to the atoms. Moreover, after about three lattice vibrations, $\sim 1 \mathrm{ps,}$ the atom motion will correspond to a Maxwellian thermal velocity distribution. ${ }^{40}$ Since the total time of energy deposition exceeds 1 ps for the higher deposited energies, and the kinetic energies are below the threshold, this kind of energy deposition can be considered to a good approximation to correspond to thermal deposition. To illustrate the correspondence of the added kinetic energy with sample heating, we carried out a test simulation with a single $10 \mathrm{eV}$ energy deposition to one atom, and analyzed the temperature in the vicinity of this atom as a function of time and distance. We found that after $0.2 \mathrm{ps}, 1 \mathrm{ps}$, and $5 \mathrm{ps}$, the average temperature increase within a $1 \mathrm{~nm}$ radius sphere from the atom was $30 \mathrm{~K}, 9 \mathrm{~K}$, and $0.9 \mathrm{~K}$, respectively.

In the heat spike events, the average nuclear energy deposition per ion was found to be $6.0 \mathrm{keV}$ (since some energy was lost to electronic stopping). Hence, the number of atoms to which the $10 \mathrm{eV}$ energy was given was chosen to be $6.0 \mathrm{keV} / 10 \mathrm{eV} \times N_{\text {ions }}$ to obtain a thermal deposition run where the nuclear energy deposition corresponded exactly to the deposition in a heat spike produced by $N_{\text {ions }}$ ions. Moreover, the time between giving atoms the energy of $10 \mathrm{eV} \Delta t$ was chosen to be $\Delta t=\Delta t_{0} /(6.0 \mathrm{keV} / 10 \mathrm{eV})$, where $\Delta t_{0}$ was the average time between impacts in the heat spike runs. In this way the time during which thermal energy was deposited also corresponded to that in heat spike runs for a given $N_{\text {ions }}$. Since there are thousands of atoms to which kinetic energy is added in random positions, the sample temperature, of course, raises strongly. Because energy is continuously dissipated away from the deposition region, which is not spherically symmetric, it is not possible to give a single value or even a position-independent function for the temperature to which the thermal energy deposition region is heated. However, the average temperature in the entire simulation cell is illustrated in Fig. 4.

For both the heat spike and thermal runs, temperature was controlled at the side and bottom boundaries of the simulation cell, and the evolution of the system was followed until it had cooled down sufficiently that no further changes in surface morphology were observed, amounting to total simulation times of 300-500 ps. For comparison with AFM experiments, the approach of an AFM tip at a regular grid interval was simulated to obtain a surface height distribution. The simulated tip radius was chosen to have about the same ratio to the crater widths as in the AFM experiments. Craters observed in the simulations for different bombarding area radii $r$ are illustrated in Fig. 5.

\section{RESULTS AND DISCUSSION}

Experimental images of surface damage in $\mathrm{Cu}$ produced by arcs obtained with the dc setup are given in Fig. 2 . These

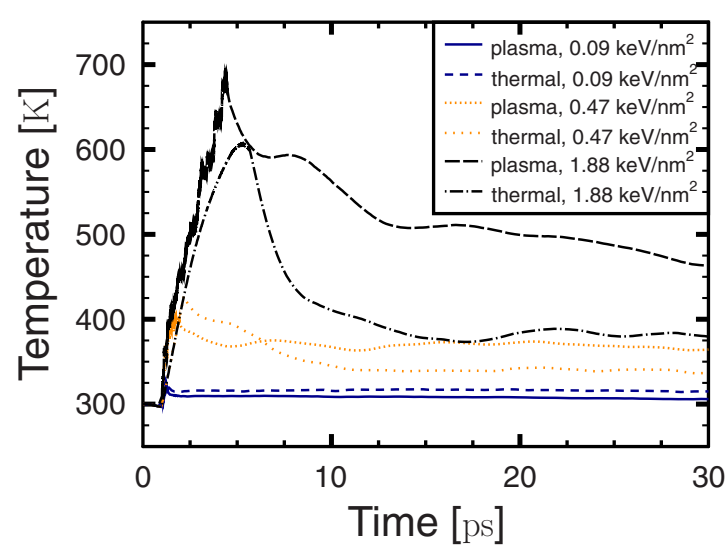

FIG. 4. (Color online) Development of the average temperature in the system for different numbers of bombarding ions. The bombardment of the surface started at $t=1 \mathrm{ps}$. Atoms that are above the original surface are not included in the temperature calculation since sputtered atoms can have high kinetic energies which do not contribute to the substrate temperature. The total number of atoms in these systems was 7 million.

images, which are very similar to typical crater images in the literature, ${ }^{3}$ show characteristic complex features such as a fingerlike structures outside the main crater rim.

To understand the formation of the craters, we first carried out PIC simulations of the buildup and time development of the plasma between two electrodes separated by the same distance as in the experiments, and using a typical experimental electric potential difference. ${ }^{32}$ The central result obtained from the PIC simulations is the formation of a sheath potential ${ }^{41}$ close to the cathode surface, where under the given conditions the electric field is roughly ten times higher than the initial gradient between the cathode and anode. Over this field, positive $\mathrm{Cu}$ ions are accelerated toward the cathode surface, obtaining potential energies peaking around $8 \mathrm{keV}$, for the external electric field value of $500 \mathrm{MV} / \mathrm{m}$. The results are illustrated in Fig. 3. These energies are typical for ion irradiation of materials, and are well known to form heat spikes that can cause massive atom redistribution and relocation, sometimes resulting in cratering. ${ }^{15,36}$ The other important result obtained from the PIC simulations is that the ion flux is very high, the total flux $\Gamma$ of both ions and neutrals over all energies being almost $\sim 10^{25}$ ions $/ \mathrm{cm}^{2} / \mathrm{s}$. This flux obtained from PIC may seem very high compared to typical ion irradiation fluxes which are 5-15 orders of magnitude lower ${ }^{42,43}$ but a simple estimate from experimental values of the typical currents in arcs on the order of $I$ $=10 \mathrm{~A}$ and final crater areas on the order of $A=(10 \mu \mathrm{m})^{2}$ (Ref. 3) gives a comparable flux of $I / e / A=6$ $\times 10^{25}$ ions $/ \mathrm{cm}^{2} / \mathrm{s}$ ( $e$ is the electron charge).

The combination of high energy and flux leads to an important insight. From studies of cascades, it is well known that the duration of a $\sim 10 \mathrm{keV}$ heat spike in $\mathrm{Cu}$ is on the order of $10 \mathrm{ps}$, and its extent in space on the order of $(5 \mathrm{~nm})^{3} \cdot{ }^{35,44}$ When these numbers are compared with the flux value, it becomes clear that during long-term bombardment of the materials with ions from the plasma, a new ion will most likely hit a heat spike area before the previous heat spike has cooled down: the mean time between impacts on a 

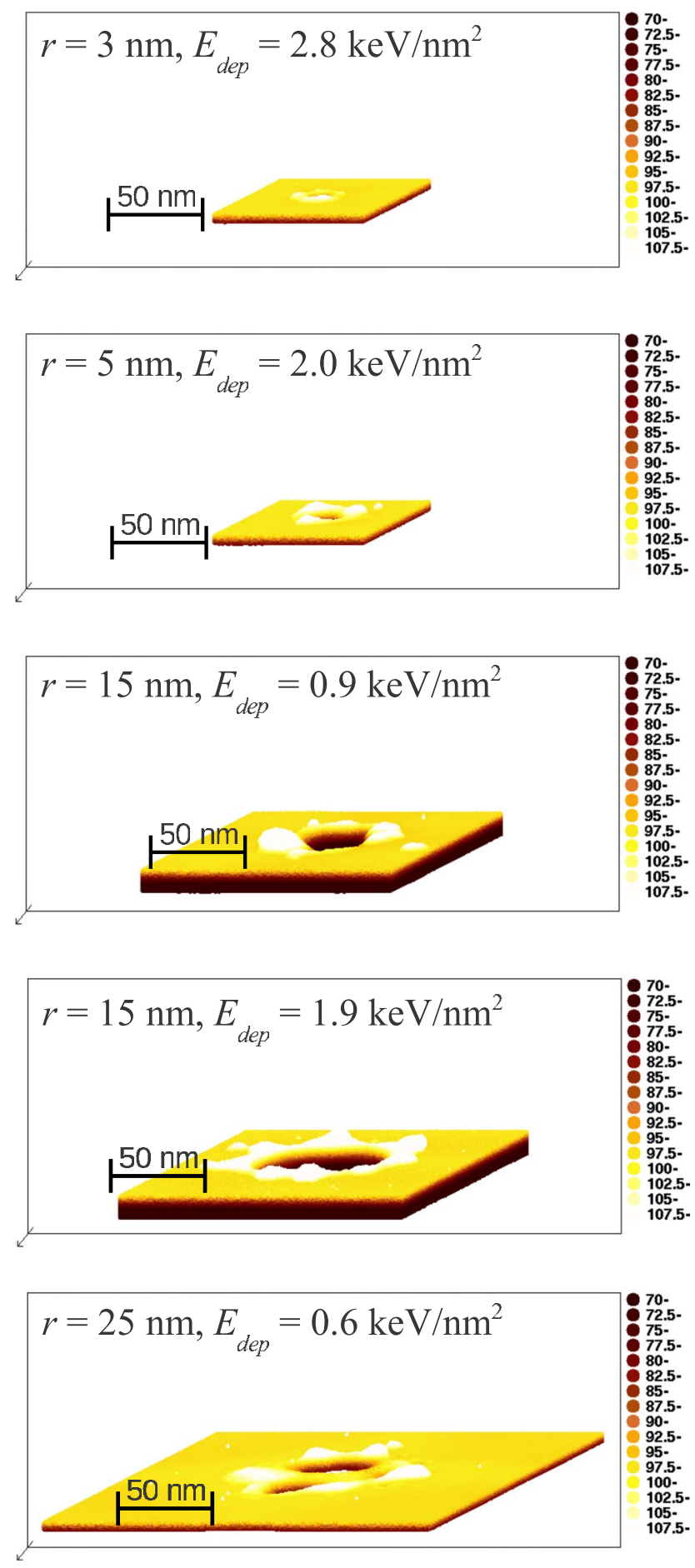

FIG. 5. (Color online) Self-similarity of craters of different size produced in the MD simulations. The simulations show craters of similar shape in the whole simulated range of $r=3-25 \mathrm{~nm}$, justifying the comparison with larger experimental craters. Only a thin slice from the top of the simulation cells is shown. The height scale is indicated on the right-hand side in units of angstrom, and both the lateral, depth and height of the plotting region is exactly the same in all frames. The frame for $r=15 \mathrm{~nm}$ and energy deposition $E_{\text {dep }}$ $=1.88 \mathrm{keV} / \mathrm{nm}^{2}$ shows the same crater as that in the left-hand side of Fig. 6. The crater for $r=25 \mathrm{~nm}$ was produced by a relatively low-energy deposition, and hence the crater is split into several parts, similar to some of the craters in Fig. 1.
$A=(5 \mathrm{~nm})^{2}$ area is $1 /(\Gamma A)=0.4 \mathrm{ps}$, i.e., much less than 10 ps.

In a single heat spike, the sample is locally extremely hot (with temperatures on the order of $10000 \mathrm{~K}$ by the time a Maxwellian energy distribution has been reached ${ }^{40}$ ) but cools down rapidly over a time scale of a few picoseconds. ${ }^{45,46}$ In the current situation, when several heat spikes overlap, the temperature in the system is a four-dimensional function of space and time. The spatial average temperature in the entire system excluding sputtered atoms is illustrated in Fig. 4. The figure shows that initially the temperature rises rapidly during the bombardment and subsequently cools down slowly due to the temperature scaling at the boundaries. The systems with higher energy deposition are naturally heated up to higher temperatures.

The MD results of arc surface damage are illustrated with snapshots in Fig. 6 (animations of the spike overlaps and surface morphology development are available in the supplementary material $\left.{ }^{31}\right)$. The end result of the overlapping heat spike conditions are craters which are very similar to those observed in experiments, see Fig. 2.

In particular, the MD simulations show the formation of long "fingers" of atoms during the dynamic evolution of the crater. These can turn around to form frozen-in fingers lying on the surface, which explains the elongated atom ridges often observed next to the craters in the experiments. Alternatively, the fingers can break up due to the Rayleigh instability, ${ }^{47}$ leading to the emission of atom clusters which can land elsewhere on the surface or be emitted into the plasma. Similar effects have been previously reported to occur in connection with cratering by single atoms and atom clusters and were found to be in good agreement with experiments. ${ }^{16,17,21,22,48,49}$ However, single $\mathrm{Cu}$ atoms at similar energies have not been previously reported to produce such complex crater features with an appreciable probability, ${ }^{36}$ and thus in the current case the effects are associated with the overlap of several heat spikes (see also analysis of fluence dependence below).

Moreover, we also observed the formation of "double" craters in the simulations that strongly resemble some of the craters observed in the experiments, compare the inset in Fig. 2 with some of the smaller craters in the experimental image (note that the ions are coming in stochastically in space and time, and thus spatial separation of craters may occur especially for lower fluences). On the other hand, also the plasma density may fluctuate, and these fluctuations may lead to a temporal variation in the incoming ion flux that could also explain overlapping craters. Further study of the plasma will be needed to establish which one of the mechanisms is more significant for complex crater formation.

Similar complex cratering was observed for the full range of impact areas simulated (circles with radii $r$ from 3 to 25 $\mathrm{nm}$ ). Thus, although the simulated craters are smaller than the experimentally observed ones, the fact that craters are observed for all simulated sizes shows that the MD simulations would likely produce craters also in the experimental size range of $\sim 0.1-10 \mu \mathrm{m} .{ }^{3}$ Unfortunately computer capacity limitations prevented simulating system sizes significantly larger than $25 \mathrm{~nm}$. We carried out systematic simulations with $r=15 \mathrm{~nm}$ for different durations of the arc, as our 


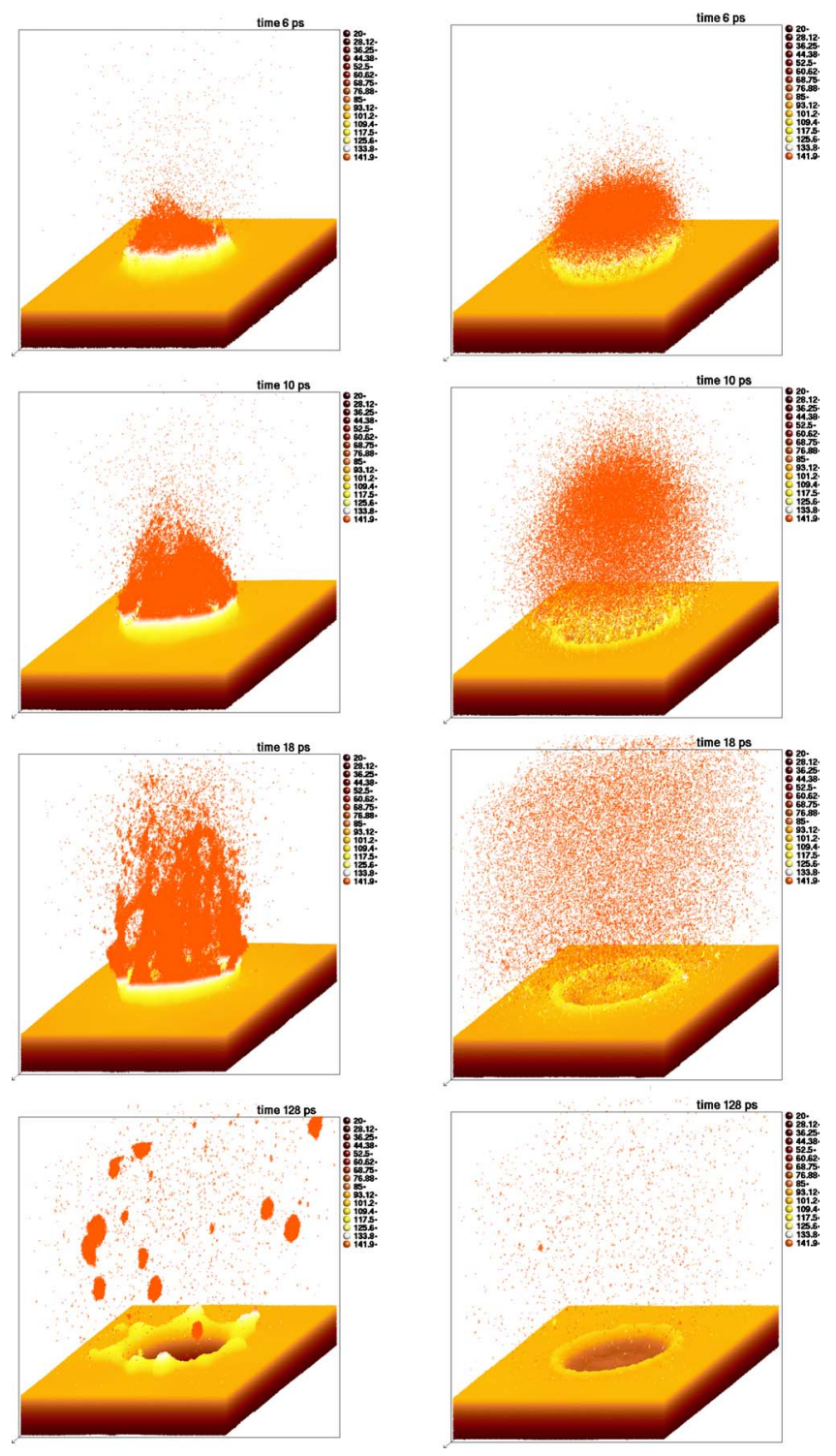

FIG. 6. (Color online) Snapshots of the time development of cratering by arcs. Left: crater produced by energetic plasma ion flux obtained from dc plasma simulations. Right: crater produced by nonballistic (thermal) energy deposition but depositing the same amount of energy as in the plasma ion case. Only a thin slice from the top of the simulation cells is shown. Each dot shows the position of an atom, due to the large number of atoms involved individual atoms cannot be distinguished at the surface. The atoms are colored according to the height above the surface, with dark yellow (medium gray in grayscale) giving the original surface position, and light yellow (light gray) and red (dark gray) positions of atoms above the surface. The height scale is given in angstrom to the right. In both cases the total-energy deposition was $1.88 \mathrm{keV} / \mathrm{nm}^{2}$. Note how in the dc case massive fingers of atoms come up, which eventually break up and lead to the formation of complex surface features and cluster emission.

PIC model was not accounting for the extinguishing of the plasma. In practice this was implemented as a different total number of bombarding ions and hence deposited energy $E_{\text {dep }}$. The results are illustrated in Fig. 7, which shows the crater rim size and amount of emitted (sputtered) atoms as a

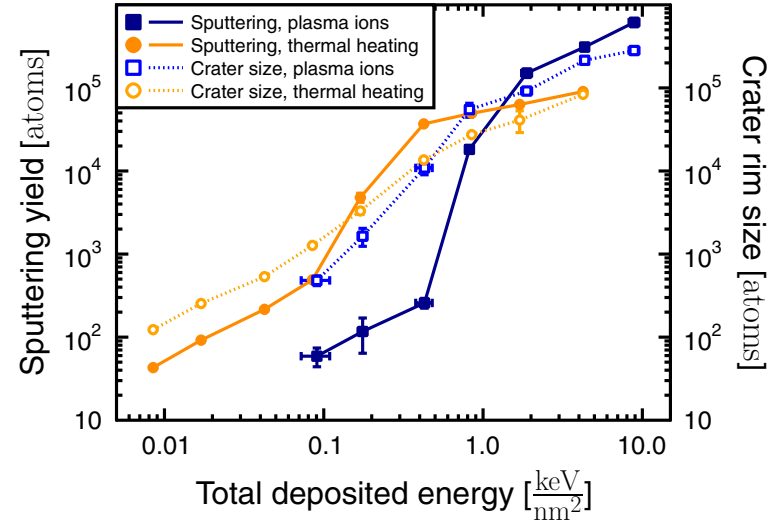

FIG. 7. (Color online) Results from MD simulations of the crater formation due to the flux of particles from the plasma for a bombarding area of $r=15 \mathrm{~nm}$. The sputtering yield signifies the total amount of particles that leave the sample because of the intense particle bombardment over the whole duration of one arcing event. The crater rim size is the total number of atoms above the surface but still connected to the sample. We also calculated the sizes of the excavated areas (crater wells), but this data is not shown for clarity, since it is almost exactly equal to the sum of the sputtering and crater rim size and thus carries no additional information. Also shown are the same quantities due to nonballistic (thermal) energy deposition that does not involve high kinetic energies.

function of the energy deposited by plasma ions on the surface. The data shows a distinct and strong transition in the crater size around a deposited energy of $E_{\text {dep }}$ $=0.8 \mathrm{keV} / \mathrm{nm}^{2}$. The reason is that below this value, the number of bombarding ions is so small that significant overlap of heat spikes has not yet occurred, and craters are produced essentially as for independent single ions. Hence, the increase is approximately linear in this region. With an increase in fluence, the overlap leads to the formation of a single molten zone covering the whole bombarded area (see also animation arcmd_liquidjoint_r15nm_N100_DC.avi in supplementary material ${ }^{\overline{3} 1}$ ), and the complex cratering mechanisms emerge due to the very large energy deposition. The threshold value of $E_{d e p}=0.8 \mathrm{keV} / \mathrm{nm}^{2}$ can, considering the maximum penetration depth of the ions $\sim 8 \mathrm{~nm}$, be shown to correspond to the energy deposition where all the material in the bombarded area is heated above the melting point. Once the full melting threshold has been achieved, additional ions only have the effect of excavating more material deeper out of the bulk. Hence, at higher deposited energies, the increase in crater size is again roughly linear.

The results for the nonballistic (thermal) energy deposition are shown in Figs. 6 and 7. The difference to the heat spike events is clear: although a crater is formed in the thermal cases, it has a much simpler morphology, which does not resemble the complex shapes seen in experiments. Moreover, Fig. 7 shows that the threshold of the dependence of sputtering yield and crater rim size with deposited energy is not as pronounced for the thermal ions. This is because in case of the thermal ions, a transition to form a large and deep fully molten region does not occur. This is further illustrated in Fig. 8, which shows that the ions with energies obtained from the plasma simulation lead to crater shapes that corre- 


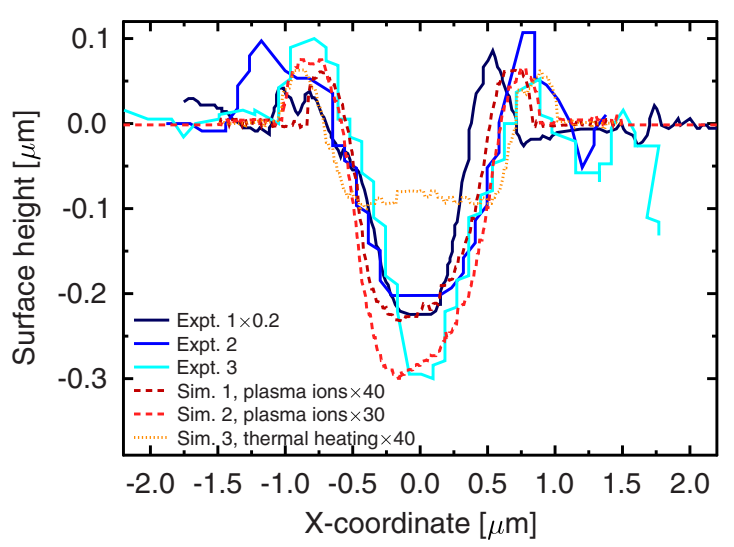

FIG. 8. (Color online) Crater shape profiles obtained from experiments and simulations. The experimental shapes were obtained from AFM measurements and the simulated ones from a simulation of a spherical AFM tip approaching the atomic coordinates obtained from the MD simulations. However, since the experimental tip radius was below $35 \mathrm{~nm}$, tip convolution effects were negligible. One of the experimental shapes has been multiplied by 0.2 to illustrate that the experimental crater shapes are self-similar over different scales. Computer capacity limitations prevented simulation of micron-sized craters, but after multiplication by a factor of 30-40, the simulated crater shapes obtained from the plasma ions agree very well with the experimental shapes. However, the nonballistic (thermal) energy deposition crater shape is clearly different from the experimental ones. Note also that in the two simulated cases of plasma bombardment, the shapes are similar with only a difference of 1.3 in the multiplication factor, although the fluence was a factor of 2.5 different.

spond very closely to the experimental ones. The thermal deposition, on the other hand, produces a clearly different shape.

We determined the aspect ratio of craters also quantitatively, defined by the rim-maximum to rim-maximum width of the crater $w$ (along the diameter), and then the depth measured from the average height of the same rim maxima to the bottom of the crater $d$. The rim maxima were used to define the width since the outside edge of the crater rims is often irregular. We found for the experimental single ion craters an aspect ratio $d / w$ of $0.26 \pm 0.04$ and for the simulated plasma ion craters for high energy depositions $\left(E_{\text {dep }} \gtrsim 1 \mathrm{keV} / \mathrm{nm}^{2}\right)$ $d / w=0.23 \pm 0.03$. The excellent agreement in the aspect ratio verifies that one possible explanation to the experimental deep crater formation is high-flux, high-fluence energetic ion bombardment of the surface.

The atoms emitted in the nonballistic (thermal) case are practically all individual atoms. Cluster connectivity analysis of the emitted atoms showed that in the heat spike cases for 100 ions or more, about $70 \%$ of the material is initially in clusters larger than ten atoms, while in the thermal energy deposition case virtually none (less than $1 \%$ ) were. The observation that the heat spike conditions lead to cluster emission is supported by recent experiments ${ }^{50}$ observing the Coulomb explosion of atom clusters in arc plasmas. It also directly supports the experimental deduction that cathode spots move around the surface in steps due to droplet emission. ${ }^{2,4}$ Since the thermal energy deposition did not lead to cluster emission, the thermal scenario is inconsistent with the experimental observation.

\section{CONCLUSIONS}

In conclusion, using a combination of experiments and computer simulations, we have shown that the huge fluxes of energetic ions can form craterlike damage on the surface and lead to sputtering of large atom clusters. The craters have a complex shape that can be explained by the strong nonequilibrium heating of the material due to energetic ions accelerated in the plasma sheath potential.

\section{ACKNOWLEDGMENTS}

We thank J. Samela for useful discussions. This work was performed within the Finnish Centre of Excellence in Computational Molecular Science (CMS), financed by The Academy of Finland and the University of Helsinki. Generous grants of computer time from the Center for Scientific Computing in Espoo, Finland, are gratefully acknowledged.
*Corresponding author; kai.nordlund@ helsinki.fi

${ }^{1}$ G. A. Mesyats and D. I. Proskurovsky, Pulsed Electrical Discharge in Vacuum (Springer, Berlin, 1989).

${ }^{2}$ B. Jüttner, Beitr. Plasmaphys. 19, 25 (1979).

${ }^{3}$ R. Behrisch, in Physics of Plasma-Wall Interactions in Controlled Fusion, NATO Advanced Studies Institute Series, Series B: Physics Vol. 131, edited by D. E. Post and R. Behrisch (Plenum Press, New York, 1986), pp. 495-513.

${ }^{4}$ B. Jüttner, J. Phys. D 34, R103 (2001).

${ }^{5}$ H. H. Braun, S. Döbert, I. Syratchev, M. T. I. Wilson, and W. Wuensch, CERN Technical Report No. CERN/PS 2002-062 (RF) (unpublished).

${ }^{6}$ G. McCracken, J. Nucl. Mater. 93-94, 3 (1980).

${ }^{7}$ J. Rager, A. Flaig, G. Schneider, T. Kaiser, F. Soldera, and F. Mücklich, Adv. Eng. Mater. 7, 633 (2005).
${ }^{8}$ A. Descoeudres, Ph.D. thesis, EPFL, 2006.

${ }^{9}$ Sputtering by Particle Bombardment I, edited by R. Behrisch (Springer, Berlin, 1981).

${ }^{10}$ A. Anders, S. Anders, M. A. Gundersen, and A. M. Martsinovskii, IEEE Trans. Plasma Sci. 23, 275 (1995).

${ }^{11}$ The surface damage is similar for both sparks and arcs, and hereinafter we use for brevity the term "arc," but all the results are relevant for both sparks and arcs.

${ }^{12}$ K. A. Holsapple and K. R. Housen, Icarus 187, 345 (2007).

${ }^{13}$ J. M. Walsh, G. L. Straddling, G. C. Idzorek, B. P. Shafer, and W. X. Do, Int. J. Impact Eng. 14, 775 (1993).

${ }^{14}$ K. L. Merkle and W. Jäger, Philos. Mag. A 44, 741 (1981).

${ }^{15}$ M. Ghaly and R. S. Averback, Phys. Rev. Lett. 72, 364 (1994).

${ }^{16}$ J. Samela and K. Nordlund, Phys. Rev. Lett. 101, 027601 (2008). 
${ }^{17}$ J. Samela and K. Nordlund, Nucl. Instrum. Methods Phys. Res. B 263, 375 (2007).

${ }^{18}$ K. Nordlund and J. Samela, Nucl. Instrum. Methods Phys. Res. B 267, 1420 (2009).

${ }^{19}$ R. M. Schmidt and K. R. Housen, Int. J. Impact Eng. 5, 543 (1987).

${ }^{20}$ A. L. Yarin, Annu. Rev. Fluid Mech. 38, 159 (2006).

${ }^{21}$ M. Ghaly, K. Nordlund, and R. S. Averback, Philos. Mag. A 79, 795 (1999).

${ }^{22}$ T. J. Colla, R. Aderjan, R. Kissel, and H. M. Urbassek, Phys. Rev. B 62, 8487 (2000).

${ }^{23}$ M. Kildemo, Nucl. Instrum. Methods Phys. Res. A 530, 596 (2004).

${ }^{24}$ A. Descoeudres, S. Calatroni, and M. Taborelli, Proceedings of 11th Biennial European Particle Accelerator Conference, EPAC '08, Genoa, Italy, 2008, p. 577 (http://accelconf.web.cern.ch/ AccelConf/e08/papers/mopp012.pdf).

${ }^{25}$ C. Birdsall and A. Langdon, Plasma Physics via Computer Simulation, 1st ed. (McGraw-Hill, New York, 1985).

${ }^{26} \mathrm{R}$. Hockney and J. W. Eastwood, Computer Simulation Using Particles, 5th ed. (IOP, Bristol and Philadelphia, 1999), Chaps. 1.1-1.5, 2.1-2.4, and 9.1-9.3.

${ }^{27}$ K. Matyash, R. Schneider, F. Taccogna, A. Hattayama, S. Longo, M. Capitelli, D. Tskhakaya, and F. X. Bronold, Contrib. Plasma Phys. 47, 595 (2007).

${ }^{28}$ S. Trajmar, W. Williams, and S. K. Srivastava, J. Phys. B 10, 3323 (1977).

${ }^{29}$ M. A. Bolorizadeh, C. J. Patton, M. B. Shah, and H. B. Gilbody, J. Phys. B 27, 175 (1994).

${ }^{30}$ A. Aubreton and M.-F. Elchinger, J. Phys. D 36, 1798 (2003).

${ }^{31}$ See supplementary material at http://link.aps.org/supplemental/ 10.1103/PhysRevB.81.184109 for supplementary information and more examples.

${ }^{32}$ H. Timko, K. Matyash, R. Schneider, F. Djurabekova, K. Nordlund, A. Hansen, A. Descoeudres, J. Kovermann, A. Grudiev, W. Wuensch, S. Calatroni, and M. Taborelli, Contrib. Plasma Phys. (to be published).

${ }^{33}$ M. P. Allen and D. J. Tildesley, Computer Simulation of Liquids
(Oxford University Press, Oxford, England, 1989).

${ }^{34}$ M. J. Sabochick and N. Q. Lam, Phys. Rev. B 43, 5243 (1991).

${ }^{35}$ K. Nordlund, M. Ghaly, R. S. Averback, M. Caturla, T. Diaz de la Rubia, and J. Tarus, Phys. Rev. B 57, 7556 (1998).

${ }^{36}$ K. Nordlund, J. Keinonen, M. Ghaly, and R. S. Averback, Nature (London) 398, 49 (1999).

${ }^{37}$ W. H. Press, S. A. Teukolsky, W. T. Vetterling, and B. P. Flannery, Numerical Recipes in C: The Art of Scientific Computing, 2nd ed. (Cambridge University Press, New York, 1995).

${ }^{38}$ K. Nordlund, Comput. Mater. Sci. 3, 448 (1995).

${ }^{39} \mathrm{P}$. Lucasson, in Fundamental Aspects of Radiation Damage in Metals, edited by M. T. Robinson and F. N. Young, Jr. (ORNL, Springfield, 1975), pp. 42-65.

${ }^{40}$ T. Diaz de la Rubia, R. S. Averback, R. Benedek, and W. E. King, Phys. Rev. Lett. 59, 1930 (1987); see also 60, 76(E) (1988).

${ }^{41}$ T. J. M. Boyd and J. J. Sanderson, The Physics of Plasmas (Cambridge University Press, Cambridge, UK, 2003).

${ }^{42}$ M. Nastasi, J. Mayer, and J. Hirvonen, Ion-Solid InteractionsFundamentals and Applications (Cambridge University Press, Cambridge, Great Britain, 1996).

${ }^{43}$ L. C. Feldman and J. W. Mayer, Fundamentals of Surface and Thin Film Analysis (North-Holland, New York, 1986).

${ }^{44}$ R. S. Averback and T. Diaz de la Rubia, in Solid State Physics, edited by H. Ehrenfest and F. Spaepen (Academic Press, New York, 1998), Vol. 51, pp. 281-402.

${ }^{45}$ H. Zhu, R. S. Averback, and M. Nastasi, Philos. Mag. A 71, 735 (1995).

${ }^{46}$ A. E. Stuchbery and E. Bezakova, Phys. Rev. Lett. 82, 3637 (1999).

${ }^{47}$ L. Rayleigh, Proc. R. Soc. London 29, 71 (1879).

${ }^{48}$ H. M. Urbassek, Nucl. Instrum. Methods Phys. Res. B 31, 541 (1988).

${ }^{49}$ K. Nordlund, J. Tarus, J. Keinonen, S. E. Donnelly, and R. C. Birtcher, Nucl. Instrum. Methods Phys. Res. B 206, 189 (2003).

${ }^{50}$ V. Ziemann, Nucl. Instrum. Methods Phys. Res. A 575, 539 (2007). 